\title{
Electron microscopy of myocardial tissue. A nine year review
}

\author{
H S Mudhar, B E Wagner, S K Suvarna
}

\begin{abstract}
Aim-To review and reassess the role of this department's experience with routine electron microscopy of myocardial tissues.

Methods-A nine year series of myocardial samples that underwent electron microscopy analysis were audited. Fifty nine samples were derived from 46 male and 13 female subjects with an age range of 15-90 years (mean, 50.6). Forty two samples were endomyocardial specimens, with 13 being derived from explanted hearts, and four from necropsies. Two cases were from transplanted hearts. These were all reviewed in a blinded fashion, by all three authors separately, in terms of the myocardium at the ultrastructural level. Subsequently, the interpretations/diagnoses were cross compared with the light microscopy and clinical data results.
\end{abstract}

Accepted for publication 20 September 2000

Department of Horthathology, Hospital, Herries Road, Sheffield

H S Mudhar B E Wagner

S K Suvarna

Correspondence to:

Dr Suvarna

s.k.suvarna@sheffield.ac.uk

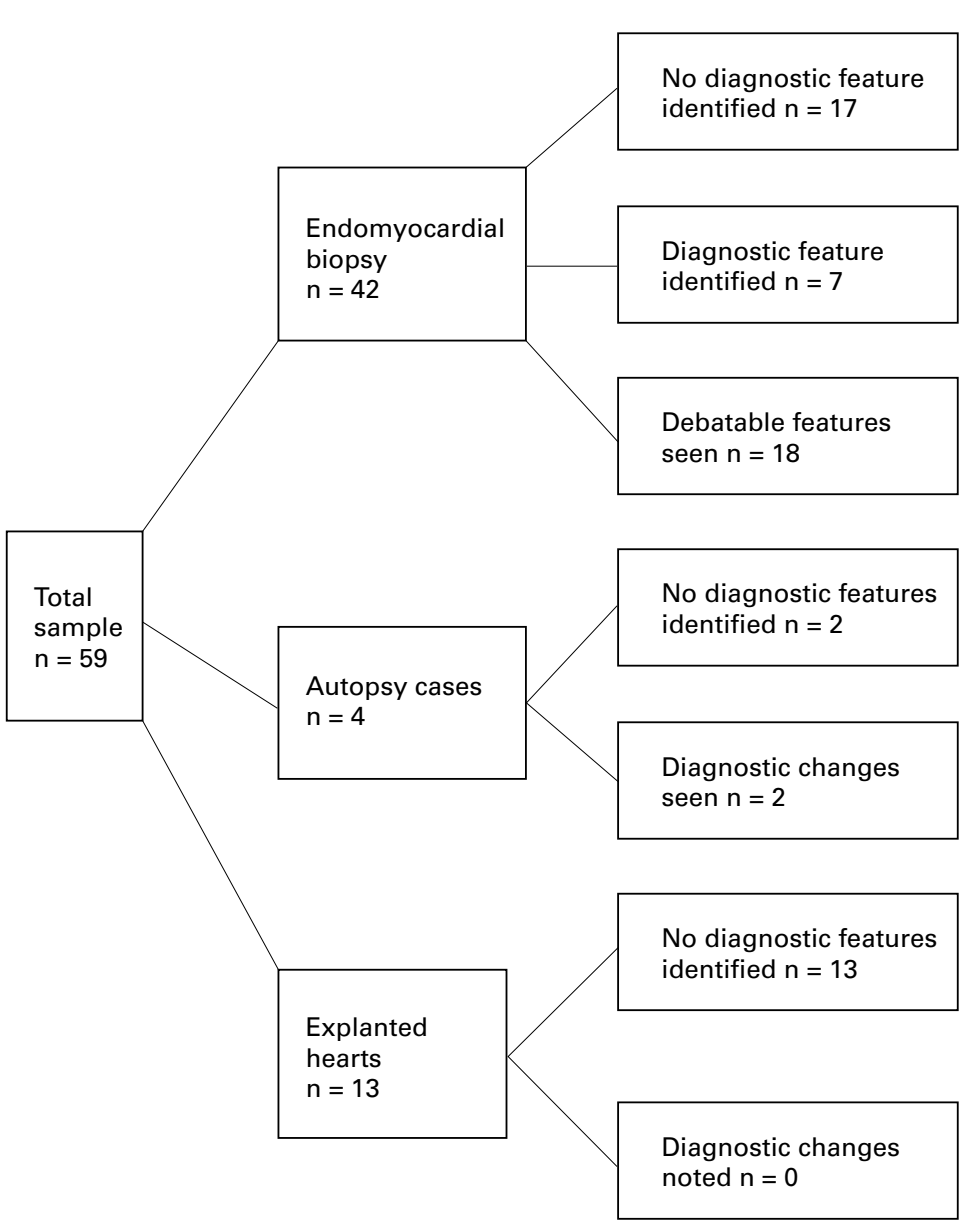

Figure 1 Tabular display of the results in terms of type of biopsy and diagnostic yield.
Results-Four cases of amyloid were identified; in addition, one case of granulomatous inflammation and one case of basophilic degeneration were seen, although all these had been evident on light microscopy. One case of possible mitochondrial myopathy was found. A total of 18 cases revealed changes of a presumed non-specific type including glycogen, lipid, and mitochondrial accumulations. Varying types of degeneration involving myofibres were seen together with variations in interstitial fibrosis and occasional cytoplasmic inclusions.

Conclusion-Overall, although interesting, the electron microscopy of myocardial tissue added little to the understanding of the patient's disease, with only one case showing changes not found at light microscopy or with other investigations. Further study might shed light on the "non-specific" ultrastructural findings encountered.

(F Clin Pathol 2001;54:321-325)

Keywords: electron microscopy; myocardial tissue; mitochondrial myopathy

Electron microscopy has been a commonly used technique within histopathology for the past 40 years. It has been valuable in the investigation of renal disease, tumour differentiation, and storage disorders, and the identification of infective agents. In addition, it has a role in the investigation of the ultrastructural detail in cases of putative cardiomyopathy/cardiac dysfunction. ${ }^{1}$ There are several well known $^{2}{ }^{3}$ commentaries on the normal myocardium at the ultrastructural level, and we have used these to evaluate samples from explanted hearts, endomyocardial biopsies, and postmortem tissues. We chose to re-evaluate our cases in terms of diagnostic yield for myocardial tissue, and to reconsider the place of electron microscopy in the investigation of myocardial disease.

\section{Methods}

All samples in which electron microscopy of the myocardium had been performed were retrieved, together with the clinical data and the light microscopy sections. The samples had originally been placed into 3\% buffered glutaraldehyde at the time of biopsy, processed into resin, and cut for transmission electron microscopy. To avoid bias, the three authors independently reviewed the ultrastructural photomicrographs without access to the light microscopy or clinical information. The analysis involved consideration of the individual 


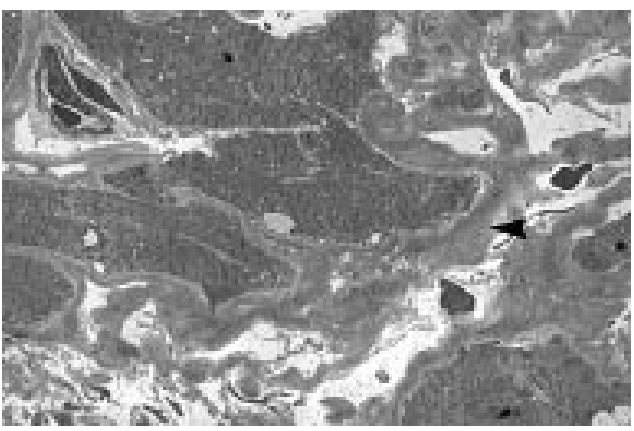

Figure 2 Amyloid (arrowhead) is seen encircling a myocyte (original magnification, $\times 1890$ ).

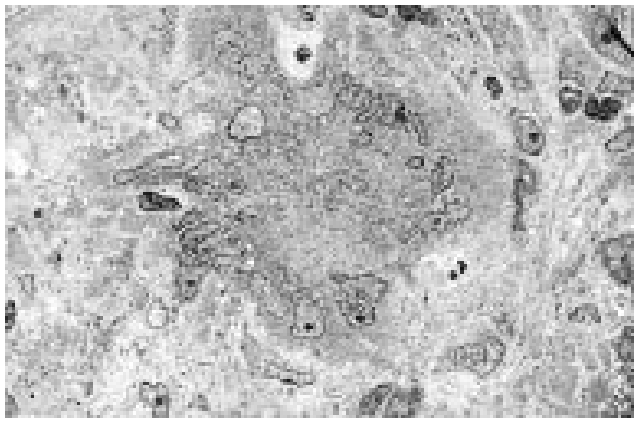

Figure 3 A Langhan's giant cell is seen within a granuloma (original magnification, $\times 1700$ ).

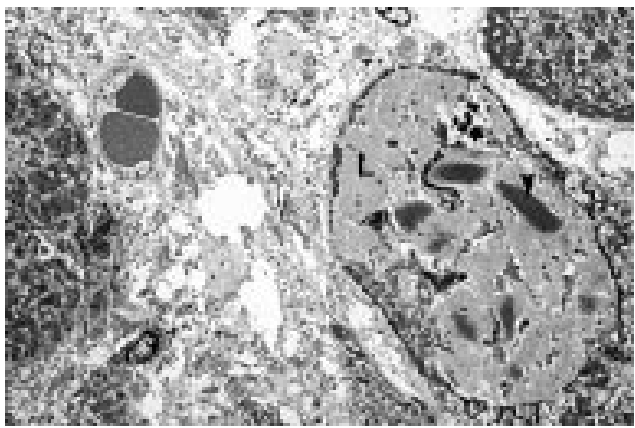

Figure 4 Basophilic degeneration showing light (L) and dark (arrowhead) zones within a myocyte (original magnification, $\times 2460$ ).

components of cardiac tissue. Thus, the interstitium, myocyte shape, cell membrane, intercalated disc, mitochondria, sarcoplasmic reticulum, glycogen load, nucleus, lysosome pattern, and any other features that were of interest were considered. The results were then collated and discussed, producing a consensus for the presence and type of abnormalities identified. These were subsequently compared with the clinical information and the light microscopy results.

\section{Results}

Over nine years, within a pool of over 1600 cardiac biopsies taken mainly for the assessment of cardiac rejection, 59 samples (46 male, 13 female) were taken for electron microscopy with a patient age range of $15-90$ years (mean, 50.6). Most (42) were endomyocardial samples, with two taken from transplant recipients. A further 13 were derived from explanted hearts and four were obtained from necropsies. Figure 1 gives a tabular breakdown of the

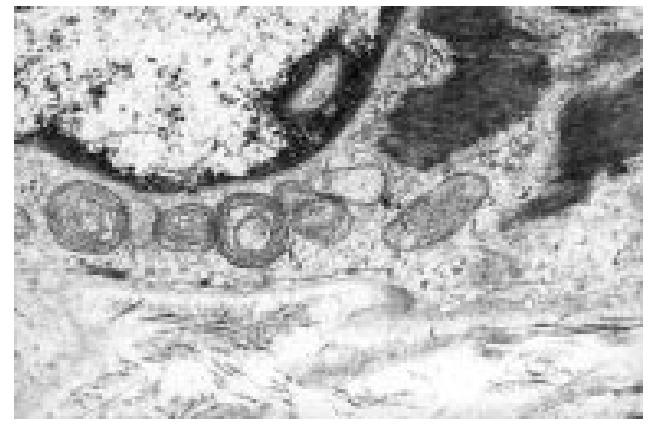

Figure 5 Mitochondria showing abnormal circular cristae within degenerate myocytes (original magnification, $\times 29500$ ).

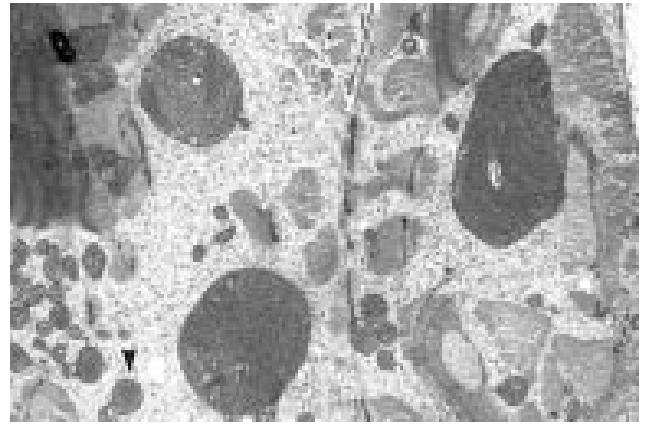

Figure 6 Pronounced variation in mitochondrial size and an abnormal cristal architecture were occasionally seen (normal mitochondrion size arrowed) (original magnification, $\times 8700$ ).

results. It was noted that minimal clinical data beyond the usual differential diagnoses of cardiomyopathies was given on request forms.

The positive cases showing specific myocardial disease included four cases of amyloid, showing the classic pericellular encasement non-branching fibrils (fig 2). All these cases were found to have Sirius/Congo red positivity on light microscopy. A single case of granulomatous myocarditis was identified in which a background of chronic inflammatory cells was seen together with an epithelioid/giant cell granuloma (fig 3). No infective agent was seen. This was from a case of probable sarcoid, diagnosed after the biopsies were taken, although the granulomatous inflammatory process and giant cell nature was evident on paraffin wax embedded sections of myocardium. Another sample showed the characteristic light and dark degenerative changes of basophilic degeneration (fig 4). A single case with abnormal myofibre degeneration and atypical mitochondria was seen in a patient with dilated cardiomyopathy, and was interpreted as a putative mitochondrial myopathy (fig 5). However, this patient recovered from his dilated, dysrhythmic status to the point where he was able to resume normal activities. Consequently, this case might be better placed with others discussed in the next paragraph.

A further 18 samples showed a variety of ultrastructural changes in which the myocardial cell architecture/background was not "normal", and in which the features provoked discussion as to their possible role in myocardial dysfunction. The changes included focal pooling of glycogen, the presence of large lipid droplets, pronounced variation in the number of mitochondria in each 


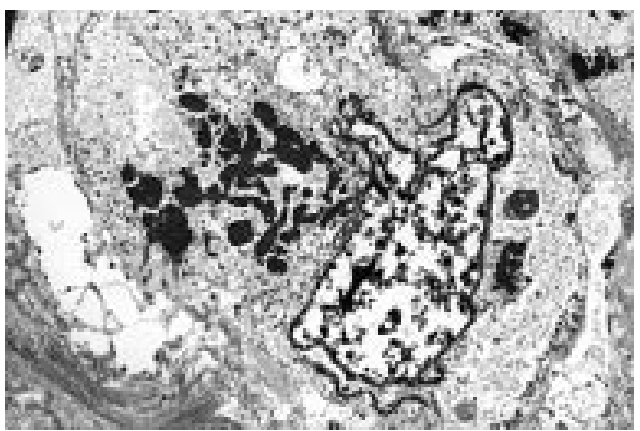

Figure 7 A cardiac myocyte showing a nemaline-like area of degeneration (original magnification, $\times 7370$ ).

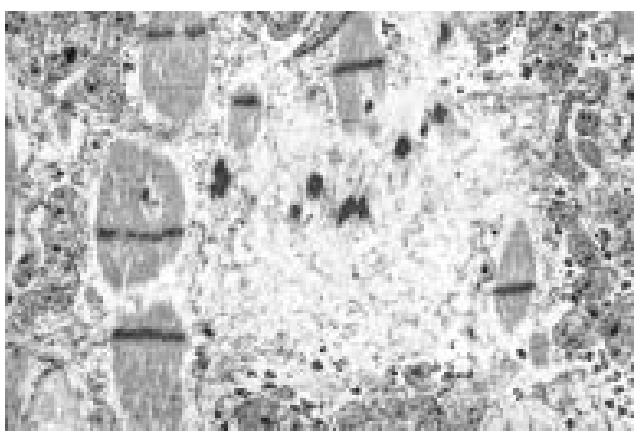

Figure 8 Focal myofibre degeneration seen in a dilated cardiomyopathy (original magnification, $\times 16$ 960).

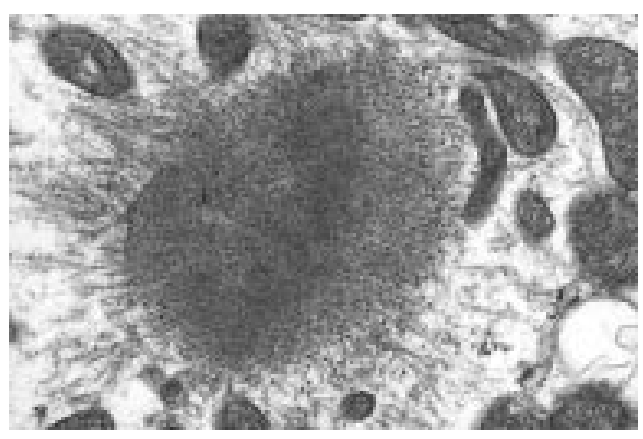

Figure 9 Cardiac myofibre disaggregation (original magnification, $\times 43$ 020).

cell, and a variable degree of fibrosis in the background interstitium. Furthermore, variations in the size of the mitochondria, focal myofibre degeneration/disaggregation, nemaline-like degeneration, and paracrystalline arrays were noted (figs 6-10). Variation in nuclear contours was often noted (fig 11). Another case had an aggregate of eosinophils, not evident on light microscopy. The remaining 34 cases were judged to be normal.

There were two cases in the postmortem group that showed gross fatty change. These samples were from an elderly woman with "tabby cat" heart and a patient with histological changes of myoclonic epilepsy with ragged red fibres (MERRF) (fig 12). The findings could be regarded as educational even in the absence of new diagnostic features being seen.

The explanted hearts studied were derived from patients with dilated cardiomyopathy undergoing transplantation. Some had a degree of ischaemic heart disease. None showed features that were of diagnostic quality in terms of identifying the aetiology of the cardiomyopathy.

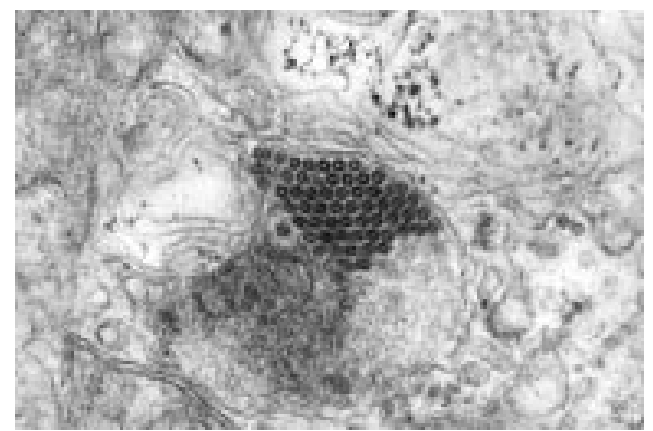

Figure 10 Paracrystalline array seen in a dilated cardiomyopathy (original magnification, $\times 150$ 000).

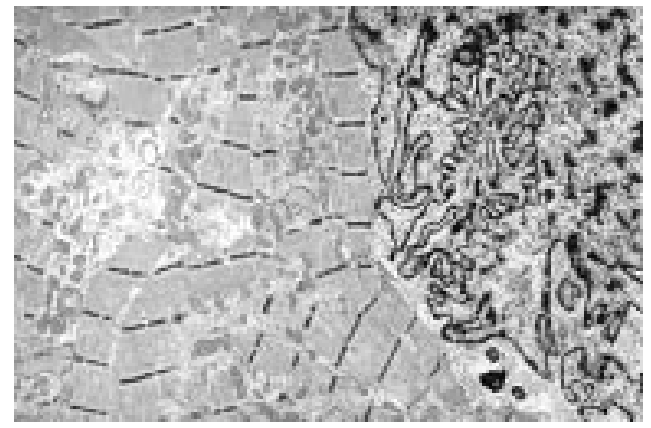

Figure 11 Hypercomplex nuclear folding, possibly in relation to microtubule contraction (original magnification, $\times 6340$ ).

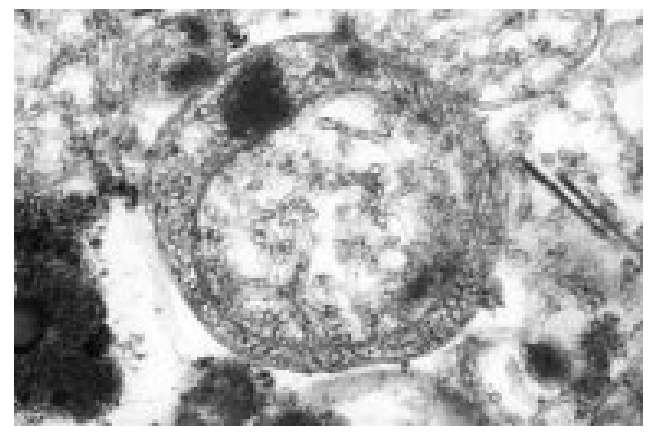

Figure 12 Mitochondrial myopathy case showing atypical mitochondrial cristae (original magnification, $\times 63$ 400).

The yield of new diagnoses from these biopsies was seven in 59 patients (12\%). Alternatively, one might consider the yield solely to be one case of debatable mitochondrial myopathy from the 59 cases $(1.7 \%)$. Conversely, this figure may be an underestimate, depending on how one views the other changes described above. If one considers the yield of changes seen from the different types of biopsy, then one can see that the postmortem group of four cases showed two with interesting features, but no new diagnoses. None of the 13 explanted hearts from cardiac transplant patients provided information in terms of a positive diagnosis as to the underlying aetiology of the patient's cardiomyopathy. The 42 endomyocardial biopsy samples showed the features of amyloid, granulomatous myocarditis, and basophilic degeneration described above as well as the "non-specific" changes.

\section{Discussion}

In most circumstances, electron microscopy is used in conjunction with light microscopy, 
immunohistochemistry, and other investigative methods to determine the pathophysiology of cardiac dysfunction. ${ }^{145}$ It may be used in the assessment of acute ischaemia/ischaemic sudden death and alcoholism. In our centre it is customary to take a minimum of four endomyocardial biopsies for light microscopy and simultaneously a sample for electron microscopy during right heart catheterisation/biopsy. Other centres have similar protocols, sometimes augmented by frozen tissue samples for immunofluorescence, enzyme assay, or genetic analysis. ${ }^{67}$ Although the routine use of electron microscopy of endomyocardial samples amounts to only $2.1 \%$ of our total electron microscopy workload, with each sample costing about $£ 25$ in consumables, it is a slow and labour intensive process. We chose to audit our experience over nine years, first to assess the yield of diagnostic data; second to compare this with the light microscopy and limited clinical data provided; and third to re-evaluate our understanding of myocardium at the ultrastructural level.

We found seven cases, all from endomyocardial biopsies and one from an explanted heart, in which there was a positive diagnosis with relevance to clinical management. However, the four cases of amyloid were all seen on conventional light microscopy with standard histochemical amyloid stains. Amyloid and other histochemical stains (Perl's, periodic acid Schiff (PAS), ABDPAS, Masson's trichrome, elastic van Giesen) are routinely performed on all our myocardial biopsies and, therefore, apart from providing confirmatory ultrastructural data, little was added by electron microscopy. The single case of granulomatous disease (subsequently diagnosed as sarcoid) was also detected at light microscopy before the ultrastructural views, as was the case of basophilic degeneration. It was perhaps useful to examine a granuloma at very high magnification within the differential diagnosis of infectious processes, but neither of these cases had their management altered. Electron microscopy was useful for identifying one case of putative mitochondrial cardiomyopathy, even though in retrospect the patient recovered. Alternatively, the changes seen point to the reason for his "temporary" cardiac disorder.

The 18 cases that showed non-specific features are worthy of consideration, despite the lack of clinical and morphological correlation. However, because the "normal" myocardium of the general population is not usually sampled for electron microscopy it is difficult to be dogmatic and state that these changes are truly abnormal. Normal cardiac ultrastructure is often portrayed in textbooks as an ideal image, with which to compare diseased tissues. However, many control/normal cardiac myocytes are invariably derived from tissue resected at congenital heart disease surgery, ${ }^{8}$ or from human postmortem material, ${ }^{9}$ both of which might be considered not to be normal. If one has not defined normality, then the non-specific features might simply reflect a range of the normal population. If so, then our study goes a little way to dealing with this deficiency. Alternatively, these features described could represent subtle or early changes, reflecting particular diagnostic entities that as yet have not been fully catalogued, with their end point being a cardiomyopathy.

It should be noted that previous studies of human myocardial tissue at the ultrastructural level have also identified some of these non-specific features in the setting of cardiomyopathies. They have included alterations in myofibre and nuclear architecture as well as mitochondrial number. ${ }^{810}$ Other studies have analysed the value of electron microscopy as a semiquantitative tool in the study of cardiomyopathy, correlating extracellular matrix and cellular myofibre values with functional myocardial studies. ${ }^{611}$ Other centres have used electron microscopy to assess apoptosis within the myocardium. ${ }^{12}{ }^{13}$ However, the data are not clear, with variations between centres as to the effectiveness of electron microscopy in this regard. ${ }^{6}{ }^{14}$ On a more experimental level, deletions of the desmin gene have been shown to cause ultrastructural changes parallelling evolution into a cardiomyopathy. ${ }^{15}$ Perhaps the most useful finding from this review exercise was the enhancement of our own understanding of the many variations seen within the context of myocardial ultrastructural detail. This was particularly so in cases with myopathic features and those with nuclear crenation. ${ }^{16}{ }^{17}$

It might be argued that we have demonstrated that electron microscopy is not cost effective in terms of the investigation of myocardial disease within our adult biased caseload. On the basis of the data presented, we would not argue with this interpretation, but it is only by gaining experience with regular exposure that we feel confident to interpret these samples. Perhaps to avoid the unnecessary duplication of diagnostic data we shall henceforth process, but not cut sections/ examine tissues at the ultrastructural level, unless we have excluded amyloid and other cardiac disorders with light microscopy. However, because we spread the cost of this investigation within the larger pool of renal biopsies at this site, from an academic perspective we propose to continue to investigate the ultrastructure of myocardial tissue. We consider that it is still a pertinent investigation in those whose diagnoses are not forthcoming from conventional methods, particularly in paediatric centres with rare cardiomyopathies such as MERFF. $^{4}{ }^{18}$

One interesting realisation upon completion of the study was that only a few cases provided any form of clinical feedback. Because we are a tertiary referral centre for cardiac disorders this is perhaps not surprising because patients often return to their referral centre. However, we would argue that this limits the understanding of myocardial pathophysiology because we have had only a limited opportunity to reconsider the tissues. The reality of this observation is that cardiologists and other clinicians will ultimately receive a less efficient service than is possible. 
We have also had the opportunity to consider the quality assurance aspect of our service. Cardiac dysfunction is a major cause of morbidity and mortality within the UK. Although smoking and ischaemic heart disease are the main causes of cardiac dysfunction, there is an important cohort of patients with primary myocardial disease that is all too often labelled as ischaemic in origin. To investigate these patients fully, biopsy sampling should be considered to augment imaging, genetic, and other tests. The question arises as to who should be examining these tissues, and with what modalities. Although centres such as ours will see myocardial tissues at electron microscopy, is it an absolute requirement, and how many does one need to see to be proficient? Should there be a form of external quality assessment within cardiac pathology?

In conclusion, despite electron microscopy having been used for a considerable time relatively few cases appeared to benefit directly from this investigation in our centre. Perhaps this reflects our case mix, with few paediatric cases being studied. Electron microscopy is relatively expensive in terms of time, machinery, and human labour, and does not produce important and diagnostic information in many cases. The inability to predict the cases in which it will be most useful requires one to either bear the cost of this technique for the bulk of the patients with non-ischaemic cardiomyopathy (also permitting experience to be maintained), or to rely upon other tests.

1 Albala A, Fenoglio JJ. Diagnostic electron microscopy of endomyocardial biopsies. In: Papamidimitrou JM, Henderson DW, Spagnolo DV, eds. Diagnostic ultrastructure of nonneoplastic diseases. Edinburgh: Churchill Livingstone, 1992:254-9.
2 Ferrans VJ, Rodriguez ER. Ultrastructure of the normal heart. In: Silver M, ed. Cardiovascular pathology, 2 nd ed. Edinburgh: Churchill Livingstone, 1991:43-129.

3 Wheater PR, Burkitt HG, Daniels VG. Functional histology. Edinburgh: Chuchill Livingstone, 1979.

4 Schaltz AA, Apitz J, Hort W, et al. Endomyocardial biopsy in infants and children: experience in 60 patients. Pediatr Cardiol 1990;11:15-21.

5 Maisch B, Bauer E, Hufnagel G, et al. The use of endomyocardial biopsy in heart failure. Eur Heart $\mathcal{F}$ 1988;9(suppl): H57-71.

6 Hammond EH, Menlove RL, Anderson JL. Predictive value of immunofluoresence and electron microscopic evaluation of endomyocardial biopsies in the diagnosis and prognosis of myocarditis and idiopathic dilated cardiomyopathy. $\mathrm{Am}$ Heart $\mathcal{F}$ 1987;114:1055-65.

7 Sidi D, Le Bidois J, Piechaud JF, et al. Enzyme activities of the mitochondrial respiratory chain in child cardiomyopathies. 34 cases prospectively studied by endomyocardial biopsy. Arch Mal Couer Vaiss 1992;85:541-6.

8 Scholz D, Diener W, Schaper J. Altered nucleus/cytoplasm relationships and degenerative structural changes in human dilated cardiomyopathy. Cardioscience 1994;5:127-38.

9 Narula J, Pandey P, Arbustini E, et al. Apoptosis in heart failure: release of cytochrome $\mathrm{c}$ from mitochondria and activation of caspase-3 in human cardiomyopathy. Proc Natl Acad Sci U S A 1999;96:7614-16.

10 Baandrup U, Florio RA, Roters F, et al. Electron microscopic investigation of endomyocardial biopsy samples in hypertrophy and cardiomyopathy. A semiquantitative study in 48 patients. Circulation 1981;63:1289-98.

11 Schaper J, Mollnau H, Hein S, et al. Interactions between cardiomyocytes and extracellular matrix in the failing human heart. $Z$ Kardiol 1995;84(suppl 4):33-8.

12 Valente $M$, Calabresse F, Thienne G, et al. In vivo evidence of apoptosis in arrhythmogenic cardiomyopathy. $A m \mathcal{F}$ Pathol 1998;152:479-84.

13 Schaper J, Lorenz-Meyer S, Suzuki K. The role of apoptosis in dilated cardiomyopathy. Herz 1999;24:219-24.

14 Frenzel H, Kasper M, Kuhn B, et al. Light and electron microscopy findings in early and late stages of heart failure. Studies of endomyocardial biopsies of patients with latent and dilated cardiomyopathy. $Z$ Kardiol 1985;74:135-43.

15 Thornell L, Carlsson L, Li Z, et al. Null mutation in desmin gene gives rise to a cardiomyopathy. $\mathrm{f} \mathrm{Mol}$ Cell Cardiol 1997;29:2107-24.

16 Ghadially FN. Ultrastructural pathology of the cell and matrix, 4th ed. Boston: Butterworth-Heinemann, 1997.

17 Papamidimitrou JM, Henderson DW, Spagnolo DV. Skeletal muscle. In: Papamidimitrou JM, Henderson DW, Spagnolo DV, eds. Diagnostic ultrastructure of non-neoplastic diseases. Edinburgh: Churchill Livingstone, 1992:595-6.

18 Rahman S, Leonard JV. Mitochondrial disorders. Curr Pediatr 1997;7:123-7. 